\title{
Visualizing particulate antigen targeting to dendritic cells (DCs) in vitro
}

\begin{abstract}
Dendritic cells (DCs) are the unique antigen presenting cells (APCs) that can bridge innate and adaptive immune responses. Their roles in antigen uptake, processing, and presentation have been upgraded by various tools and techniques of biomedical research. The current article describes how the targeting of particulates by bone marrow-derived DCs (BMDCs) can be observed in vitro. This issue has been addressed by using flow cytometry and confocal microscopy. Elaborating these mechanisms will help understand the targeting activities of DCs in vitro and in vivo.
\end{abstract}

Keywords: Antigen uptake, Micro particle, Particulate, Phagocytosis, Pinocytosis, Presentation, targeting
Volume I Issue 2 - 2014

\author{
Ghimire TR \\ Department of Veterinary and Primate Health, Global Primate \\ Network, Nepal \\ Correspondence: Tirth Raj Ghimire, Department of \\ Veterinary and Primate Health, Global Primate Network, \\ Kathmandu, Nepal, Tel 00 977। 4334784, \\ Email tirtha@primatelife.org
}

Received: September 17, 2014 | Published: October 25, 2014
Abbreviations: APC, Antigen Presenting Cell; BMDC, Bone Marrow-derived Dendritic Cell; BTS, Biotyramide System; DAPI, 4', 6-diamidino-2-phenylindole; DCs, Dendritic Cells; EaGFP, Ealpha Green Fluorescence Protein; FITC, Fluorescein Isothiocyanate; FSC, Forward Scatter Characteristics; HRP, Horse Radish Peroxidise; MP, Micro Particle; pMHC, peptide Major Histocompatibility Complex II; PE, Phycoerythrin; SSC, Side Scatter Characteristics

\section{Introduction}

DCs are central to the induction of adaptive immunity through their unique ability to activate naive T cells. ${ }^{1}$ To initiate an adaptive immune response, a number of signals are required in naive $\mathrm{T}$ cells. Among these signals, signal 1 is the cognate signal provided by peptide: major histocompatibility II (p:MHCII) complexes expressed on DC. ${ }^{2,3}$ To provide this signal, DCs internalize an exogenous protein antigen, process it into peptides, load these onto MHC molecules and export these complexes on the DC surface. ${ }^{4-6}$ Interestingly, DCs are 'voracious eaters' during their lifetime, feeding on soluble antigens by macropinocytosis and particulate antigens derived from particulate adjuvants and dying or apoptotic cells, and microorganisms from their immature to mature age by phagocytosis..$^{7-10}$ Importantly, phagocytosis involves triggering of cell surface receptors that drive act in polymerization and active internalization of particles. ${ }^{11}$

While DC has been shown to be an efficient antigen sampling cell, its antigen targeting effect has been subject of interests especially due to its significance in the reduction of antigens required to stimulate an effective $\mathrm{T}$ cell response. Because antigen formulated in mineral adjuvants, micro particles, and nanoparticles is modified into particulate form and is well-targeted by DCs, these additives are the important therapeutic targets such as drugs and vaccines. These additives are able to act as an intracellular antigen depot providing a prolonged antigen presentation due to sustained release. ${ }^{12,13}$ Understanding how these additives will affect the function of DCs will be subject of interests for drug and vaccine designers. Thus, this study has been conducted to observe and analyze the role of BMDCs in taking up a soluble and particulate antigen and their efficiency in presentation in vitro.

In this study, Ealpha green fluorescence protein (EaGFP)/YAe system was used to visualize antigen uptake and presentation by BMDCs $^{12,14}$ (Figure 1). This system allows assessment of antigen uptake/degradation and, in combination with the YAe antibody antigen presentation in situ. ${ }^{12,14,15}$ When this antigen is internalized by DCs, EaGFP is degraded and the E $\alpha$ peptide is presented by IAbMHCII on the cell surface. These E $\alpha$ : IAbMHCII complexes can be detected by staining the cells with YAe antibody because this antibody can efficiently bind the complex of $\mathrm{E}_{\alpha(52-68)}$ with IAbMHCII. ${ }^{14,16}$ Therefore, the YAe antibody sees what $\mathrm{T}$ cell receptor sees (Figure 1). In this assay, GFP signal inside cell suggests the antigen engulfed within cell and missing of this signal indicates its complete degradation. Thus, this direct, simple, and easy technique was used in the observation of phagocytosis and pinocytosis and presentation of soluble antigen by BMDCs in vitro.

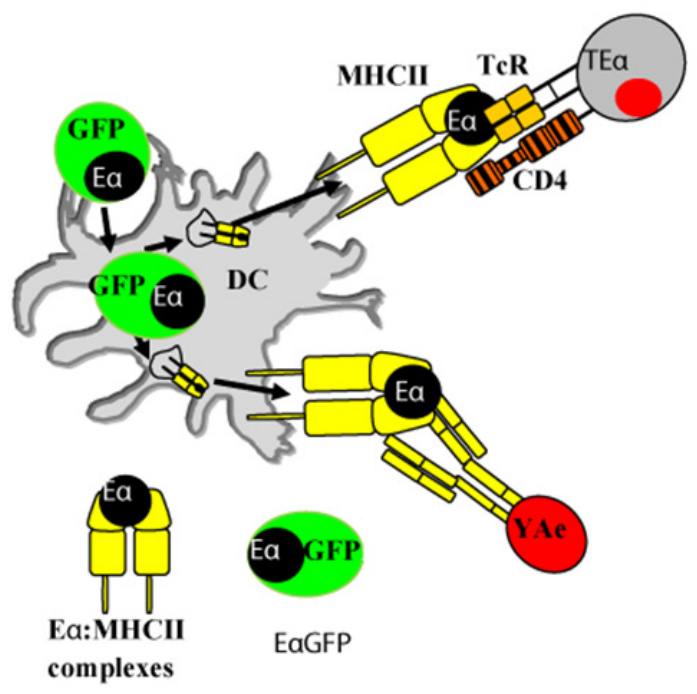

Figure I The EaGFP:YAe System: The YAe antibody sees what the TCR sees. This antibody binds $\mathrm{E} \alpha(52-68)$ : MHCll complexes presented by DCs. GFP signal represents the antigen accumulating cells whereas $Y A e$ positive signal represents antigen presenting cells in the context of E $\alpha$ IAbMHCII complexes.

\section{Materials and methods}

Preparation of DCs from murine bone marrow: marrow cells $(2 \times 106 /$ well $)$ from six- to eight-week-old C57BL/6 $(\mathrm{H}-2 \mathrm{~b} / \mathrm{b})$ mice were used to prepare BMDCs using $10 \%$ GMCSF (63x cell line supernatants) supplemented with RPMI [RPMI 1640 (Sigma, UK), $10 \%$ FCS (Gibco, UK), 100 $\mathrm{g} / \mathrm{mL}$ penicillin and streptomycin 
(Invitrogen, UK) and $100 \mu \mathrm{g} / \mathrm{mL}$ l-glutamate (Invitrogen, UK) as described previously. ${ }^{12,17}$

Antigens: The fluorescent antigen, EaGFP, was prepared using methods described previously. ${ }^{12,15}$ EaGFP is a chimeric fluorescent protein antigen ${ }^{15}$ produced from a genetic fusion of $\mathrm{E} \alpha$ peptide to GFP. ${ }^{18} \mathrm{E} \alpha$ peptide (amino acid residues 52-68) is the immune dominant epitope derived from IEd alpha chain and presented by IAb-restricted MHC class II molecules. . $^{14,16,19}$

Particulate beads uptake by DCs: To study phagocytosis of bead and antigen targeting to BMDCs, cells were incubated in media, in the Phycoerythrin (PE)-conjugated carboxylate-modified microspheres $(2 \mu \mathrm{m})(2 \mu \mathrm{L}$ beads) (Invitrogen), Bead-Mix [E $\alpha \mathrm{GFP}$ and Phycoerythrin (PE)-conjugated beads; $2 \mu \mathrm{L}$ beads containing $2.5 \times 106$ beads per $0.5 \times 106 \mathrm{DCs} /$ well], or EaGFP $(50 \mu \mathrm{g} / \mathrm{mL})$ alone for 1 hour or 24 hours and were analyzed by flow cytometry.

Cells were collected in 5mL FACS tubes (BD FALCON, BD Biosciences Discovery Lab Ware) and washed (400xg, 5minutes, $\left.4^{\circ} \mathrm{C}\right)$ in FACS buffer (5\% FCS, $0.1 \%$ sodium azide) and incubated with either purified anti-mouse CD16/CD32 (1 in 100 dilution) or $100 \mu \mathrm{L}$ Fc block (2.4G2 hybridoma supernatant) for 30minutes to prevent non-specific binding via Fc receptors. Then, cells were stained with fluorophore-labeled antibody (biotinalyted anti-mouse E $\alpha 52$ 68, eBiosciences, clone: eBioYAe, cat no. 13-5741, bio anti-mouse IgG2b, Southern Biotech, clone: A-1, cat. 0104-08, allophycocyanin (APC) streptavidin, eBiosciences, cat no. 17-4317), and incubated for 30 minutes. Cells were washed with FACS buffer twice (400xg, 5 minutes, $4^{\circ} \mathrm{C}$ ) and analyzed on a FACS Caliber (BD Biosciences). The results of flow cytometry were analyzed by FlowJo software (FlowJo 8.7.1, Stanford University 1995-96).

Similarly, some cells were incubated with beads, Bead-Mix or EaGFP in chamber slides (LabTek) for 1hour and 24hours and processed for biotyramide system (BTS). Then, cells in chamber slides were washed with Fc block, Avidin block, Biotin block (Vector Laboratories, Inc. Burlingame, CA) and stained with YAe-Bio at 1:100 dilution (Biotin conjugated anti-mouse $\mathrm{E}_{\alpha 52-68}$ peptide, YAe-bio, $0.5 \mathrm{mg} /$ $\mathrm{mL}$, eBiosciences) diluted in TNB Blocking Buffer (PerkinElmer Life Sciences). The cells were stained with Streptavidin-horse radish peroxidise (HRP) (1.2mL, PerkinElmer Life Sciences, Inc. Boston, MA, US) at the 1:100 dilution in TNB Blocking Buffer, then with biotyramide (diluted in 1:50 in Amplification Buffer, Molecular Probes, Invitrogen) and finally with Streptavidin AF 647 (Alexa Fluor 647 conjugated/tagged, Invitrogen). Finally, one drop of 4', 6-diamidino2-phenylindole (DAPI) (Vector laboratories, Inc. Burlingame, CA) was used in each well and $25 \times 60 \mathrm{~mm}$ coverslip (Borosilicate Glass, VWR International) was used. Cells were observed by confocal microscopy (LSM 510 software, Carl Zeiss). Images were analyzed by Velocity Software (Velocity 4.1, Improvision, Viscount Centre, University of Warwick Science Park, and Coventry).

\section{Results}

Antigen uptake and presenting assay by flow cytometry: Flow cytometry was used to evaluate the morphologic and cellular characteristics on the basis of the Forward Scatter Characteristics (FSC) and Side Scatter Characteristics (SSC). Cells were studied by relative size based on FSC and relative granularity based on SSC. There was no difference in FSC or SSC in cells incubated in bead or bead-mix compared in cells incubated in media or EaGFP (Figure 2). However, cells that captured beads were easy to detect due to high signals of PE at various series in flow cytometry histograms. Pinocytic activity of cells was calculated by analyzing the $\%$ of cells that have
GFP signals in Fluorescein Isothiocyanate (FITC) background. Similarly, phagocytic activity was calculated by analyzing the \% of cells that have PE-conjugated beads (Figure 2).

In this study, the efficiency of BMDCs in pinocytosis and phagocytosis was compared at 1 hour and 24hours following incubation with E $\alpha \mathrm{GFP}$ or bead, or bead-mix (Figure 2). Interestingly, BMDCs were highly efficient in phagocytosis of beads and this efficiency was increased with respect to duration of incubation (Supplementary Figure 1A). The data also showed that both \% of GFP-positive cells (Supplementary Figure 1B) and \% of YAe-positive cells ( 9 fold increase) were increased from 1 hour to 24 hours indicating BMDCs efficiently pinocytose soluble antigens and present them in

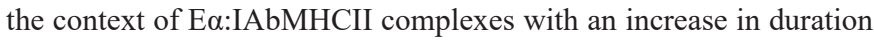
of incubation. However, total GFP-positive cells (Supplementary Figure 1B) and cells that contain both phagocytozed beads and GFP (Supplementary Figure 1C) were very low in the Bead-Mix treatment group at 1 hour as well as 24 hours. However, cells that contain both phagocytozed beads and YAe molecules sharply increased from 1hour to 24hours compared with YAe molecules in EaGFP-treated cells (Supplementary Figure 1D). These results suggest that BMDCs efficiently engulf MPs and soluble antigens and enhances processing of soluble antigens in the presence of MPs; however further a pulse chase assay should be conducted to validate this hypothesis.

Phagocytosis and antigen presenting assay by confocal microscopy: Next, BTS and confocal microscopy were used to visualize antigen capturing and antigen presenting efficiencies of BMDCs (Figure 3). These figures confirm that DAPI, PE-labeled beads, and E $\alpha \mathrm{GFP}$ are highly effective materials in visualizing antigen engulfed by BMDCs. Clear indications of more than 20 beads in some DCs were observed indicating some DCs are highly phagocytic or voracious upon MPs (Figure 3A and 3B). With the help of appropriate staining, presentation of antigen by BMDCs could be observed (Figure 3C). Overall, the data indicate that biotyramide assay and EaGFP/YAe assays could be directly and reliably used to observe particulate capturing behavior and antigen presenting characteristics of BMDCs in vitro.

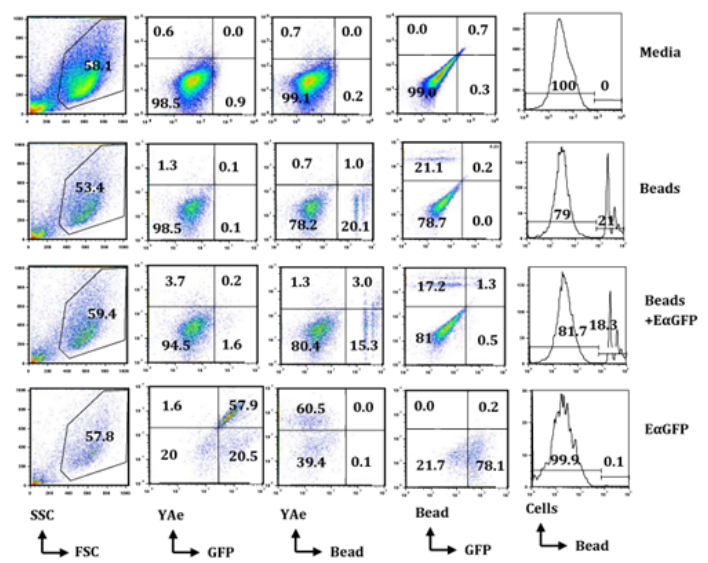

Figure 2 BMDCs are efficient in uptake of soluble antigen as well as particulate such as bead (MP) and in presentation of soluble antigen in vitro: $\mathrm{BMDCs}(0.5 \times 106 / 5 \mathrm{~mL})$ obtained from $\mathrm{C} 57 \mathrm{BL} / 6$ mice were

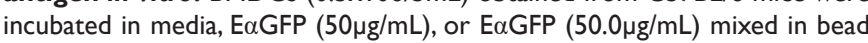
for Ihour and 24hours. Cells were stained with YAe-bio antibody and APC Streptavidin and processed for flow cytometry analysis. A total of 50,000 cells were collected based on FSC and SSC. Dot plot shows the proportion of cells positive for YAe vs. GFP, YAe vs. bead and GFP vs. bead. Histogram plots show the \% maximum of cells positive for PE-conjugated beads (MPs). All these figures are the representative diagrams obtained by analysis of cells incubated for 24hours. 


\section{Discussion and conclusion}

The use of flow cytometry and confocal microscopy in the characterization of various particles and their roles in antigen internalization by APCs have been already discussed. ${ }^{20-26}$ In contrast to these studies, the current study employs YAe/EaGFP system to detect unprocessed or native antigens in the form of GFP and processed and

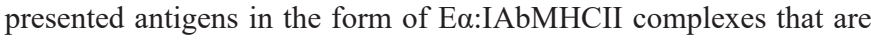
recognized by YAe antibody. For this system, flow cytometry is crucial. Flow cytometry is an exceptional method to detect, quantify, and characterize MPs in physiologic and in pathologic conditions. ${ }^{26}$ The current study shows that MPs reduced an uptake of soluble antigens and enhanced the presentation of soluble antigen with respect to time. This result may be attributed to an enhanced antigen processing efficiency of the current $2 \mu \mathrm{m}$ MPs, however it is difficult to generalize in the absence of a pulse chase experiment. Previous reports have shown that compared to $1 \mu \mathrm{m}$ particle, only $20 \mathrm{~nm}$ particles reduced antigen degradation via interference in the lysosomal compartment. ${ }^{23}$ In an in vivo experiment, negatively charged $20 \mathrm{~nm}$ particles were efficient in enhancing antigen presentation to $\mathrm{CD} 4+\mathrm{T}$ cells in lung-draining lymph nodes. ${ }^{21}$ However, glycine-coated $50 \mathrm{~nm}$ particles down regulated the stimulatory property of $\mathrm{CD} 11 \mathrm{~b}+\mathrm{DCs}$ in lung draining lymph nodes to OVA-specific T cells. ${ }^{27}$ Brewer and colleagues have shown that compared to antigen prepared in small vesicles $(<200 \mathrm{~nm})$, antigen prepared in large vesicles $(200 \mathrm{~nm}-560 \mathrm{~nm})$ tends to target early phagosomes and generated an enhanced $\mathrm{T}$ cell activation via MHCII pathway. ${ }^{20}$ This suggests the crucial role of particle sizes on antigen degradation and consequently on DC function. All these different results might be due to dissimilar particles, their sizes, functional groups and charges on these particles, APC subsets, and environment of the study performed (in vitro vs in vivo). Thus, a pulse chase experiment and flow cytometry analysis will be a beautiful model of the prediction of antigen uptake, enzymatic degradation, and antigen presentation. In this context, the E $\alpha$ GFP/YAe system and MP uptake protocol will assist to elaborate the lifecycle of particulate antigen inside DCs. ${ }^{12}$

(A)

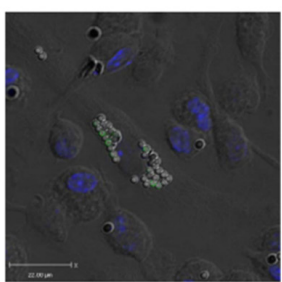

(B)

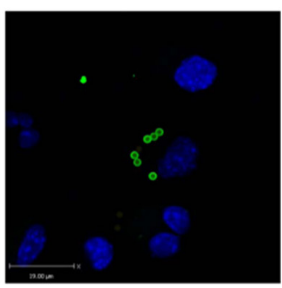

(C)

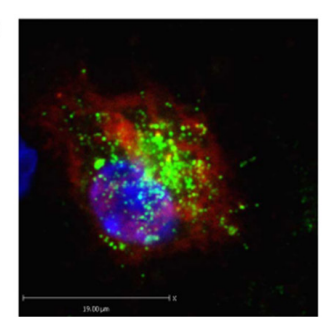

Figure 3 Particulates (MPs) target antigen to BMDCs in vitro: BMDCs were treated with E $\alpha$ GFP and or 2 micron beads and incubated for Ihour in chamber slides. Biotyramide assay was used for confocal microscopy. (A) Shows BMDCs one of which has voraciously engulfed beads (MPs). Some of these beads still have GFP signals. Nucleus is stained by DAPI that excites at $405 \mathrm{~nm}$ and gives blue color. (B) Shows some BMDCs one of which is voracious feeder on bead-mix. This cell also contains E $\alpha$ GFP-nonconjugated bead inside it. (C) Represents the BMDC that has pinocytosed soluble E $\alpha$ GFP antigen and presented it in the context of E $\alpha: I A b M H C l l$ complexes as detected by $Y A e$ antibody. YAe signals (red signal) are amplified due to binding of Streptavidin 647 which excites at far red.

Interestingly, confocal data suggest the voraciousness of BMDCs in vitro. These cells efficiently captured particulates and this efficiency increased with time suggesting the consistence voraciousness of these cells. Confocal microscopy uses BTS that includes of the use of HRP to catalyze the remains of the biotin-labeled tyramide (amplification reagent). It is crucial to note that BTS efficiently enhances both chromogenic and fluorescent signals and therefore has been a beautiful tool in the visualization of uptake of both particulate and soluble antigen and their presentation on cell surface.

In conclusion, drug delivery using particulates coupled with $\mathrm{E} \alpha \mathrm{GFP} / \mathrm{YAe}$ protocol for flow cytometry and confocal microscopy will be effective to study the antigen sampling and antigen presenting characteristics of BMDCs in the presence or absence of particles in vitro. This cellular pathway of BMDCs can be targeted to devise a novel approach for the rational design of drugs and vaccines in future.

\section{Acknowledgements}

This work was conducted in the University of Strathclyde. I acknowledge Professor J. Brewer for providing me laboratory facilities and supervision.

\section{Conflict of interest}

The author declares no financial or commercial conflict of interest. This work was conducted in the University of Strathclyde, Scotland. For this work, the author has no relation with the current affiliation as shown in the first page.

\section{References}

1. Steinman RM. Dendritic cells in vivo: a key target for a new vaccine science. Immunity. 2008;29(3):319-324.

2. Mueller DL, Jenkins MK, Schwartz RH. Clonal expansion versus functional clonal inactivation: a costimulatory signalling pathway determines the outcome of T cell antigen receptor occupancy. Annu Rev Immunol. 1989;17:445-480.

3. Mueller DL, Jenkins MK, Schwartz RH. An accessory cell-derived costimulatory signal acts independently of protein kinase $\mathrm{C}$ activation to allow $\mathrm{T}$ cell proliferation and prevent the induction of unresponsiveness. J Immunol. 1989;142(8):2617-2628.

4. Watts C. Capture and processing of exogenous antigens for presentation on MHC molecules. Annu Rev Immunol. 1997;115:821-850.

5. Watts C. Antigen processing in the endocytic compartment. Curr Opin Immunol. 2001;13(1):26-31.

6. Nelson CA, Vidavsky I, Viner NJ, et al. Amino-terminal trimming of peptides for presentation on major histocompatibility complex class II molecules. Proc Natl Acad Sci USA. 1997;94(2):628-633.

7. Trombetta ES, Ebersold M, Garrett W, et al. Activation of lysosomal function during dendritic cell maturation. Science. 2003;299(5611):1400-1403.

8. Trombetta ES, Mellman I. Cell biology of antigen processing in vitro and in vivo. Annu Rev Immunol. 2005;23:975-1028.

9. Platt CD, Ma JK, Chalouni C, et al. Mature dendritic cells use endocytic receptors to capture and present antigens. Proc Natl Acad Sci USA. 2010;107(9):4287-4292. 
10. Morefield GL, Sokolovska A, Jiang D, et al. Role of aluminumcontaining adjuvants in antigen internalization by dendritic cells in vitro. Vaccine. 2005;23(13):1588-1595.

11. Travaglione S, Falzano L, Fabbri A, et al. Epithelial cells and expression of the phagocytic marker CD68: scavenging of apoptotic bodies following Rho activation. Toxicol $<e m>$ In vitro $</ e m>$. 2002;16(4):405-411.

12. Ghimire TR, Benson RA, Garside P, et al. Alum increases antigen uptake, reduces antigen degradation and sustains antigen presentation by DCs in vitro. Immunol Lett. 2012;147(1-2):55-62.

13. Fredriksen BN, Grip J. PLGA/PLA micro- and nanoparticle formulations serve as antigen depots and induce elevated humoral responses after immunization of Atlantic salmon (Salmo salar L.). Vaccine. 2012;30(3):656-667.

14. Rudensky A, Preston-Hurlburt P, Hong SC, et al. Sequence analysis of peptides bound to MHC class II molecules. Nature. 1991;353(6345):622-627.

15. Rush CM, Brewer JM. Tracking dendritic cells in vivo. Methods Mol Biol. 2010;626:169-185.

16. Rudensky A, Preston-Hurlburt P, al-Ramadi BK, et al. Truncation variants of peptides isolated from MHC class II molecules suggest sequence motifs. Nature. 1992;359(6394):429-431.

17. Lutz MB, Kukutsch N, Ogilvie AL, et al. An advanced culture method for generating large quantities of highly pure dendritic cells from mouse bone marrow. J Immunol Methods. 1999;223(1):77-92.

18. Tsien RY. The green fluorescent protein. Annu Rev Biochem. 1998;67:509-544.

19. Rudensky A, Rath S, Preston-Hurlburt P, et al. On the complexity of self. Nature. 1991;353(6345):660-662.
20. Brewer JM, Pollock KG, Tetley L, et al. Vesicle size influences the trafficking, processing, and presentation of antigens in lipid vesicles. $J$ Immunol. 2004;173(10):6143-6150.

21. Blank F, Stumbles PA, Seydoux E, et al. Size-dependent uptake of particles by pulmonary antigen-presenting cell populations and trafficking to regional lymph nodes. Am J Respir Cell Mol Biol. 2013;49(1):67-77.

22. Kempf M, Mandal B, Jilek S, et al. Improved stimulation of human dendritic cells by receptor engagement with surface-modified micro particles. J Drug Target. 2003;11(1):11-18.

23. Seydoux E, Rothen-Rutishauser B, Nita IM, et al. Size-dependent accumulation of particles in lysosomes modulates dendritic cell function through impaired antigen degradation. Int $J$ Nanomedicine. 2014;9:3885-3902.

24. Leclerc L, Boudard D, Pourchez J, et al. Quantification of micro sized fluorescent particles phagocytosis to a better knowledge of toxicity mechanisms. Inhal Toxicol. 2010;122(13):1091-1100.

25. Orozco AF, Lewis DE. Flow cytometric analysis of circulating micro particles in plasma. Cytometry A. 2010;77(6):502-514.

26. Nielsen $\mathrm{MH}$, Beck-Nielsen $\mathrm{H}$, Andersen $\mathrm{MN}$, et al. A flow cytometric method for characterization of circulating cell-derived micro particles in plasma. J Extracell Vesicles 3. 2014

27. Hardy CL, LeMasurier JS, Belz GT, et al. Inert 50-nm polystyrene nanoparticles that modify pulmonary dendritic cell function and inhibit allergic airway inflammation. J Immunol. 2012;188(3):1431-1441. 